\title{
Assessment of biochemical markers in the early post-burn period for predicting acute kidney injury and mortality in patients with major burn injury: comparison of serum creatinine, serum cystatin- $C$, plasma and urine neutrophil gelatinase-associated lipocalin
}

Hyeong Tae Yang ${ }^{1,3}$, Haejun Yim', Yong Suk Cho ${ }^{1}$, Dohern Kym¹, Jun Hur', Jong Hyun Kim', Wook Chun ${ }^{1}$ and Hyun Soo Kim ${ }^{2 *}$

\begin{abstract}
Introduction: The reported mortality rates range from $28 \%$ to $100 \%$ in burn patients who develop acute kidney injury (AKI) and from 50\% to 100\% among such patients treated with renal replacement therapy. Recently, the serum cystatin $C$ and plasma and urine neutrophil gelatinase-associated lipocalin (NGAL) levels have been introduced as early biomarkers for AKl; the levels of these biomarkers are known to increase 24 to 48 hours before the serum creatinine levels increase. In this study, we aimed to estimate the diagnostic utility of the cystatin C and plasma and urine NGAL levels in the early post-burn period as biomarkers for predicting AKI and mortality in patients with major burn injuries.

Methods: From May 2011 to July 2012, 90 consecutive patients with a burn wound area comprising $\geq 20 \%$ of the total body surface area (TBSA) were enrolled in this study. Whole blood and urine samples were obtained for measuring the serum creatinine, serum cystatin C, and urine and plasma NGAL levels at $0,3,6,12,24$, and 48 hours after admission. Receiver operating characteristic curve, area under the curve, and multivariate logistic regression analyses were performed to assess the predictive values of these biomarkers for AKI and mortality.

Results: In the multivariate logistic regression analysis, all variables, including age, percentage TBSA burned, sex, inhalation injury, and serum creatinine levels, serum cystatin C levels, and plasma and urine NGAL levels were independently associated with AKI development. Moreover, age, sex, percentage TBSA burned, and plasma and urine NGAL levels were independently associated with mortality. However, inhalation injury and the serum creatinine and cystatin C levels were not independently associated with mortality.
\end{abstract}

Conclusions: Massively burned patients who maintained high plasma and urine NGAL levels until 12 hours after admission were at the risk of developing early AKI and early mortality with burn shock. However, the plasma and urine NGAL levels in the early post-burn period failed to predict late AKI and non-burn shock mortality in this study. Nevertheless, the plasma and urine NGAL levels were independently associated with AKI development and mortality within 48 hours after admission.

\footnotetext{
* Correspondence: hskim0901@empas.com

2Department of Laboratory Medicine, Hallym University Dongtan Sacred Heart Hospital, Hallym University College of Medicine, 7, Keunjaebong-gil, Hwaseong-si, Gyeonggi-do 445-170, Korea

Full list of author information is available at the end of the article
} 


\section{Introduction}

The reported incidence rates of acute kidney injury (AKI) among burn patients range from $<1$ to $36 \%$, depending on the population studied and the classification criteria used [1]. The reported mortality rates among burn patients who develop AKI range from 73 to $100 \%$ [2]. During the early phase of a major burn injury, the most common cause of AKI is ischemic organ damage from effective hypovolemia caused by a massive systemic inflammatory response. During the late phase of a major burn injury, sepsis and nephrotoxic agents are the most common causes of AKI.

Laboratory research has revealed that early intervention may be essential for preventing the pathophysiologic events that lead to AKI. However, serum creatinine, which is one of the main AKI biomarkers used in clinical settings, is a late marker for reduced glomerular filtration rate, which limits its use in early AKI detection and clinical therapeutic studies [3]. Recently, the levels of serum cystatin $\mathrm{C}$ and plasma and urine neutrophil gelatinaseassociated lipocalin (NGAL) have been suggested as early biomarkers for AKI as their levels were found to increase 24 to $48 \mathrm{~h}$ prior to an increase in the serum creatinine level [4-12].

Few studies have elucidated the associations of the serum cystatin C, plasma NGAL, and urine NGAL levels with AKI in patients with major burn injuries. This study had the following aims: (1) to investigate the levels of serum creatinine, cystatin $\mathrm{C}$, and plasma and urine NGAL over time during the early post-burn period; (2) to estimate the diagnostic utility of the cystatin $\mathrm{C}$ and plasma and urine NGAL levels for predicting AKI and mortality in patients with major burn injuries; and (3) to determine the relationships between the levels of these biomarkers and the burn sizes and inhalation injuries, which are known prognostic factors for burn injuries.

\section{Materials and methods}

\section{Patient selection and subgroups}

From May 2011 to July 2012, 90 consecutive patients were enrolled in this prospective cohort study. The study protocol was approved by the Institutional Review Boards of Hangang Sacred Heart Hospital (IRB number 2011-143), and informed consent was obtained from all subjects. None of the patients were lost to follow up. The inclusion criteria were as follows: patients aged $\geq 18$ years with $\%$ total body surface area (TBSA) burned $\geq 20 \%$. Because we intended to investigate changes in biomarker levels during the early post-burn period, we limited the inclusion criteria to those patients who were admitted to our burn intensive care unit within $6 \mathrm{~h}$ of injury. Patients with known cardiac disease (for example, prior history of heart failure, arrhythmia, or coronary heart disease), prior kidney transplant, end-stage kidney disease, or chronic liver disease (for example, prior history of liver cirrhosis or chronic hepatitis) were excluded. Multiple data, including sex, age, body weight, presence of comorbidities, \% TBSA burned, \% TBSA with third-degree burn wounds, cause of burn injury, and presence of inhalation injury, were collected for each patient.

AKI diagnoses were made according to the risk, injury, failure, sustained loss, and end-stage kidney disease (RIFLE) criteria [13]. These criteria include an increase in the serum creatinine level $\geq 50 \%$ over the baseline or reduction in the urine output to $<0.5 \mathrm{~mL} / \mathrm{kg} / \mathrm{h}$ for a period $>6 \mathrm{~h}$. The baseline renal function was defined as the lowest known serum creatinine value during the preceding 3 months; however, most of the patients had not undergone a previous laboratory evaluation. For patients without known prior serum creatinine levels, we used the lowest serum creatinine level measured during the admission period only when the level was within a normal range. However, the lowest serum creatinine levels were abnormal in six patients. In these cases, the baseline serum creatinine levels were estimated using the modified diet in renal disease equation for the assessment of kidney function while assuming a glomerular filtration rate of $75 \mathrm{~mL} /$ minute/ $1.73 \mathrm{~m}^{2}$ [13]. Considering the patients' ages, it is possible that we underestimated the AKI. However, all six patients were included in the AKI group with a RIFLE stage R. Therefore, AKI was not underestimated because of the baseline serum creatinine levels.

As described in the Introduction, the primary cause of AKI differs according to the time of AKI development. Thus, we classified the patients into the following three groups according to the time of AKI development: the no-AKI group; the early-AKI group, wherein AKI developed within 5 days of burn injury; and the late-AKI group, wherein AKI developed after 5 days post burn injury. The mean time from burn injury to early AKI development was 1.2 days. No patients developed AKI on day 4; however, one patient developed AKI on day 5 because of rhabdomyolysis caused by an electrical injury. Therefore, this patient was included in the early AKI patient group. Thus, early AKI was considered as AKI that developed up to 5 days after burn injury.

Additionally, we classified the patients as survivors or non-survivors according to mortality. The non-survivors with burn shock had experienced the most severe burn injuries, and their length of hospital stay was very short. Therefore, we decided to divide the patient groups into the following three groups based on the time of death: the early-death group, the late-death group, and the survival group. The early-death group included non-survivors who developed burn shock despite fluid resuscitation and died within 3 days of burn injury.

To determine whether the biomarker levels were affected by the burn size or presence of inhalation injury, 
we also classified the patients into two groups: smaller burn-size group (\% TBSA burned, 20 to $49 \%$ ) and larger burn-size group (\% TBSA burned, 50 to 100\%).

\section{Specimen collection and measurement of biomarkers}

Blood samples were collected via a central venous catheter and urine samples were collected via a Foley catheter, at 0 , $3,6,12,24$, and $48 \mathrm{~h}$ after admission to the burn intensive care unit. The blood NGAL levels were measured with the Triage NGAL reagent and Triage Meter (Alere Healthcare, San Diego, CA, USA). The serum cystatin C levels were measured according to a turbidimetric immunoassay method with the HiSense cystatin $\mathrm{C}$ kit ( $\mathrm{HBi}$, Anyang, Korea) and Hitachi 7600 analyzer (Hitachi, Tokyo, Japan). The serum and urine creatinine levels were measured according to an enzymatic method with the Cica Creatinine reagent (KANTO Chemical, Tokyo, Japan) and Hitachi 7600 analyzer (Hitachi, Tokyo, Japan).

For the urine NGAL analysis, urine specimens were transferred to centrifuge tubes and centrifuged at a relative centrifugal force $\geq 400$ for a minimum of 5 minutes; the supernatants were stored at $-70^{\circ} \mathrm{C}$ prior to batch analysis. After thawing, the specimens were mixed and centrifuged at 2,500 to $3,000 \times \mathrm{g}$ for 10 minutes prior to use, to remove any particulate matter and ensure consistency in the results. The urine NGAL levels were measured in a chemiluminescence immunoassay with an Architect i2000SR analyzer (Abbott Diagnostics, Abbott Park, IL, USA) and a dedicated urine NGAL reagent (Abbott Diagnostics). All measurements were performed according to the manufacturers' instructions.

\section{Data analysis}

Data were expressed as mean \pm SD or number (\%) for each subgroup of AKI development and mortality (Tables 1 and 2). Statistical differences among subgroups were analyzed using the Mann-Whitney $U$-test or Kruskal-Wallis test. We investigated and compared changes in serum creatinine, cystatin $\mathrm{C}$, and plasma and urine NGAL levels during the $48 \mathrm{~h}$ after admission in the groups classified according to the time of AKI development and mortality (Figures 1 and 2). Thereafter, comparisons of the mean serum creatinine, cystatin $C$, plasma NGAL, and urine NGAL levels at each point between patients with and without AKI and between survivors and non-survivors were performed and statistically analyzed. We also compared the changes in the levels of these biomarkers between the groups classified according to the burn size and inhalation injury, which are known prognostic factors for burn injury (Figures 3 and 4). The diagnostic abilities of the plasma and urine NGAL levels for predicting AKI and mortality were assessed by calculating the areas under the receiver operating characteristic curves (AUC-ROC) and the cut-off value was defined by Youden's index (Tables 3 and 4). Multivariate analysis was performed to identify variables

Table 1 Patient characteristics according to acute kidney injury (AKI) development

\begin{tabular}{|c|c|c|c|c|}
\hline & \multirow[t]{2}{*}{ Non-AKI $(n=35)$} & \multicolumn{2}{|l|}{ AKI $(n=55)$} & \multirow[t]{2}{*}{$P$-value } \\
\hline & & Early AKI $(n=31)$ & Late AKI $(n=24)$ & \\
\hline Age, mean $\pm S D$ & $45.4 \pm 14.8$ & $51.3 \pm 15.5$ & $52.5 \pm 12.4$ & 0.129 \\
\hline Sex, male:female, n (\%) & $29: 6(82: 18)$ & $28: 3(90: 10)$ & 20:4 (83:17) & 0.641 \\
\hline Mode, FB:SB:EB:CB:SKB, n (\%) & 27:1:4:2:1 (77:3:12:5:3) & 28:1:2:0:0 (91:3:6:0:0) & 20:1:1:1:1 (84:4:4:4:4) & \\
\hline TBSA burned, $\%$, mean \pm SD & $38.4 \pm 14.1$ & $70.7 \pm 22.0$ & $61.5 \pm 19.2$ & $<0.001$ \\
\hline Third-degree burn wound, $\%$, mean \pm SD & $24.6 \pm 10.2$ & $62.5 \pm 28.6$ & $48.5 \pm 21.3$ & $<0.001$ \\
\hline Inhalation injury, n (\%) & $4(11.4)$ & $17(54.8)$ & $8(33.3)$ & 0.01 \\
\hline Mechanical ventilation, n (\%) & $5(14.3)$ & $28(90.3)$ & $19(79.2)$ & $<0.001$ \\
\hline Rhabdomyolysis, n (\%) & $7(20.0)$ & $24(77.4)$ & $4(16.7)$ & $<0.001$ \\
\hline Early vasopressor, n (\%) & $1(2.8)$ & $21(67.7)$ & $3(12.5)$ & $<0.001$ \\
\hline Post-burn day of AKI, days, mean \pm SD & & $1.2 \pm 0.6$ & $18.9 \pm 12.1$ & $<0.001$ \\
\hline CRRT, n (\%) & & $7(22.6)$ & $15(62.5)$ & $<0.001$ \\
\hline Duration of CRRT, days, mean \pm SD & & $1.9 \pm 0.7$ & $10.9 \pm 2.8$ & $<0.001$ \\
\hline Sepsis, n (\%) & $3(8.6)$ & $7(22.6)$ & $20(83.8)$ & $<0.001$ \\
\hline LOS, day, mean $\pm S D$ & $55.7 \pm 23.1$ & $26.6 \pm 43.6$ & $42.2 \pm 35.2$ & 0.003 \\
\hline Mortality, n (\%) & $2(5.7)$ & $24(77.4)$ & $14(58.3)$ & $<0.001$ \\
\hline Maximal RIFLE score, n (R:l:F) & & $0: 10: 21$ & $1: 5: 18$ & \\
\hline
\end{tabular}

Early AKI, AKI that developed within 5 days of injury; Late AKI, AKI that developed after 5 days post injury; FB, flame burn; SB, scalding burn; EB, electrical burn; $C B$, chemical burn; SKB, spark burn; TBSA, total body surface area; Early vasopressor, vasopressor infusion due to shock within 1 day of admission; CRRT, continuous renal replacement therapy; LOS, length of hospital stay; RIFLE, risk, injury, failure, sustained loss, and end-stage kidney disease; $n$, number of patients. 
Table 2 Patient characteristics according to mortality

\begin{tabular}{|c|c|c|c|c|c|}
\hline & \multirow[t]{2}{*}{ Survivors $(n=50)$} & \multicolumn{3}{|c|}{ Non-survivors $(n=40)$} & \multirow{2}{*}{$\begin{array}{l}\text { Total patients } \\
(\mathrm{n}=90)\end{array}$} \\
\hline & & $\begin{array}{l}\text { Early deaths } \\
(n=17)\end{array}$ & $\begin{array}{l}\text { Late deaths } \\
(n=23)\end{array}$ & $\begin{array}{l}\text { Total deaths } \\
(n=40)\end{array}$ & \\
\hline Age, mean $\pm S D$ & $45.4 \pm 14.2$ & $54.5 \pm 13.3$ & $54.1 \pm 14.5$ & $54.3 \pm 13.8$ & $49.3 \pm 13.5$ \\
\hline Sex, male:female (\%) & $43: 7(86: 14)$ & $15: 2(88: 12)$ & 19:4 (83:17) & $34: 6(85: 15)$ & 77:13 (86:14) \\
\hline Mode, FB:SB:EB:CB:SKB (\%) & 40:1:5:3:1 (80:2:10:6:2) & 15:1:1:0:0 (88:6:6:0:0) & 20:1:1:0:1 (87:4:4:0:4) & $35: 2: 2: 0: 1(88: 5: 5: 0: 3)$ & $75: 3: 7: 3: 2(83: 3: 8: 3: 2)$ \\
\hline TBSA burned, mean $\% \pm S D$ & $41.9 \pm 15.2$ & $82.9 \pm 17.4$ & $65.7 \pm 18.4$ & $73.0 \pm 19.7$ & $55.7 \pm 23.2$ \\
\hline Third-degree burn wound, mean $\% \pm S D$ & $28.0 \pm 13.4$ & $77.0 \pm 24.2$ & $54.4 \pm 22.3$ & $64.0 \pm 25.5$ & $44.0 \pm 26.5$ \\
\hline Inhalation injury, n (\%) & $11(22)$ & $13(76.4)$ & $5(21.7)$ & $18(45)$ & $29(32.2)$ \\
\hline Mechanical ventilation, n (\%) & $12(24)$ & $17(100)$ & $23(100)$ & $40(100)$ & $52(57.8)$ \\
\hline Rhabdomyolysis, n (\%) & $10(20)$ & $15(88.2)$ & $10(43.4)$ & $25(62.5)$ & $35(38.9)$ \\
\hline AKI, n (\%) & $17(34)$ & $17(100)$ & $21(91)$ & $38(95)$ & $55(61.1)$ \\
\hline Early AKI, n (\%) (\%) & $7(14)$ & $17(100)$ & $6(26)$ & $23(57.5)$ & $31(33.3)$ \\
\hline Post-burn day of AKI, days, mean \pm SD & $14.0 \pm 15.9$ & $1.0 \pm 0.0$ & $11.2 \pm 9.8$ & $6.6 \pm 8.9$ & $9.1 \pm 12.1$ \\
\hline Cause of AKI, BS:RD:SS:MD, n & 4:3:0:10 & 16:1:0:0 & 3:3:14:1 & 19:4:14:1 & 23:7:14:11 \\
\hline CRRT, n (\%) & $5(10)$ & $0(0)$ & $17(73.9)$ & $17(42.5)$ & $22(24.4)$ \\
\hline RIFLE (R:I:F) starting CRRT, $\mathrm{n}$ & $0: 0: 5$ & & $2: 8: 7$ & $2: 8: 7$ & $2: 8: 12$ \\
\hline Post-burn day of CRRT, days, mean \pm SD & $10.8 \pm 7.6$ & & $14.9 \pm 9.9$ & $14.9 \pm 9.9$ & $14.0 \pm 9.4$ \\
\hline Duration of CRRT, days, mean \pm SD & $9.2 \pm 4.4$ & & $7.0 \pm 10.4$ & $7.0 \pm 10.4$ & $7.5 \pm 9.3$ \\
\hline Early vasopressor, n (\%) & $1(2)$ & $17(100)$ & $7(30.4)$ & $24(60)$ & $25(27.8)$ \\
\hline Vasopressor, n (\%) & $7(14)$ & $17(100)$ & $23(100)$ & $40(100)$ & $47(52.2)$ \\
\hline Sepsis, n (\%) & $10(20)$ & $0(0)$ & $20(86.9)$ & $20(50)$ & $30(33.3)$ \\
\hline Cultured organism in blood (P:A:M:O:N), $n$ & 3:4:1:1:1 & & 13:3:0:1:3 & 13:3:0:1:3 & $16: 7: 1: 2: 4$ \\
\hline Post-burn day of sepsis, days, mean \pm SD & $17.5 \pm 13.9$ & & $13.0 \pm 8.6$ & $13.0 \pm 8.6$ & $14.5 \pm 10.6$ \\
\hline $\mathrm{LOS}$, days, mean $\pm \mathrm{SD}$ & $66.0 \pm 28.2$ & $2.5 \pm 1.1$ & $19.8 \pm 15.1$ & $12.4 \pm 14.3$ & $42.2 \pm 35.2$ \\
\hline
\end{tabular}

Early deaths, non-survivors who developed burn shock in spite of fluid resuscitation and died within 3 days of burn injury; Late deaths, mortality caused by other than burn shock; FB, flame burn; SB, scalding burn; EB, electrical burn; CB, chemical burn; SKB, spark burn; AKI: acute kidney injury; Early AKI, AKI that developed within 5 day of injury; BS, burn shcok; RD, rhabdomyolysis; SS, septic shock; MD, medication; TBSA, total body surface area: CRRT, continuous renal replacement therapy; RIFLE, risk, injury, failure, sustained loss, and end-stage kidney disease; RIFLE (R:I:F), patient RIFLE criteria for patients starting CRRT; Early vasopressor, vasopressor infusion due to shock within 1 day of admission; Cultured organism: organism cultured in blood from paitents with sepsis; $\mathrm{P}$ : Pseudomonas aeruginosa; A: Acinetobacter baumanii; M: multi-resistant Staphylococcus aureus; O: Other organisms; N: blood culture negative; LOS, length of hospital stay.

independently associated with AKI development and mortality (Table 5). SPSS 17.0 software (SPSS, Inc, Chicago, IL, USA) was used for statistical analysis and a $P$-value $<$ 0.05 was considered statistically significant.

\section{Results}

\section{Patient characteristics}

The patients' demographic and characteristic data, classified according to AKI development, are listed in Table 1. Of these patients, 35 did not develop AKI, 31 were classified into the early AKI group, and 24 were classified into the late AKI group. There were 22 patients diagnosed according to creatinine criteria alone, 2 were diagnosed according to urinary criteria alone, and 26 were diagnosed according to both creatinine and urinary criteria.

The sex and age were not significantly different among the groups. However, the mean \% TBSA burned, presence of inhalation injury, mechanical ventilation, rhabdomyolysis, early vasopressor infusion, sepsis development, length of hospital stay, and mortality differed significantly between the groups. The early AKI group exhibited the most severe burn injuries, whereas the burn injuries were more severe in the late AKI group compared to the non-AKI group.

Table 2 shows the patients' demographic and characteristic data classified according to mortality. There were 50 survivors and 40 non-survivors. Among the 40 non-survivors, 17 died from burn shock within 3 days of burn injury. Patients with early deaths exhibited the most severe burn injuries, with a mean \% TBSA burned of $82.9 \%$ and a mean length of hospital stay of only 2.5 days.

\section{Changes in the levels of four biomarkers during $48 \mathrm{~h}$ after admission with respect to AKI development} The changes in the levels of each biomarker (serum creatinine, serum cystatin C, plasma NGAL, and urine NGAL) 


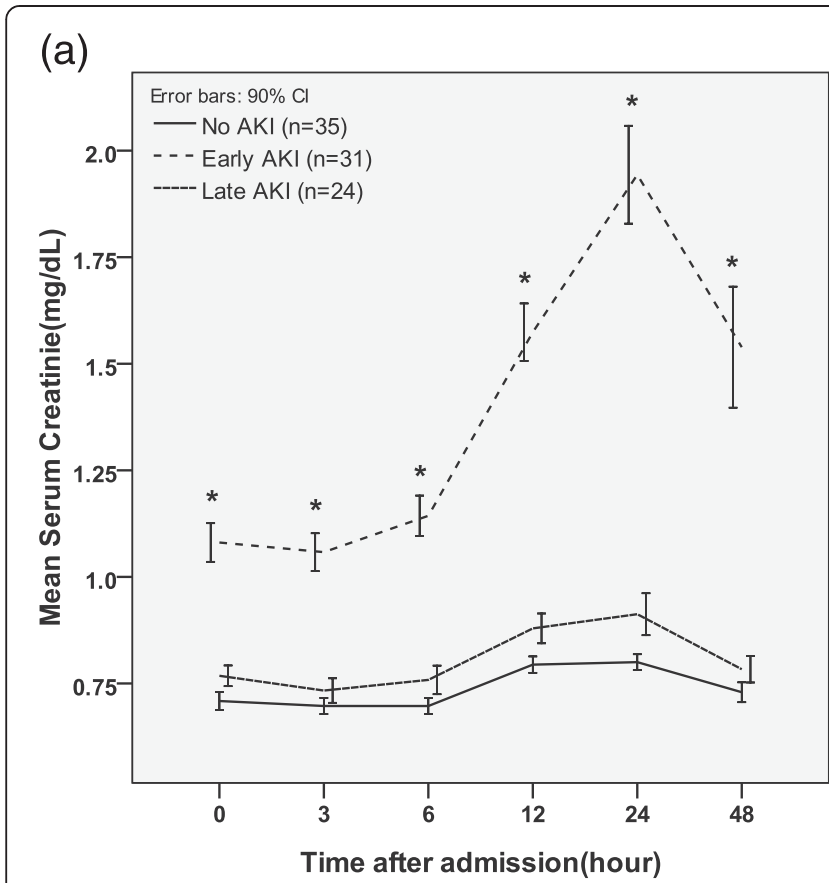

(b)

(c)
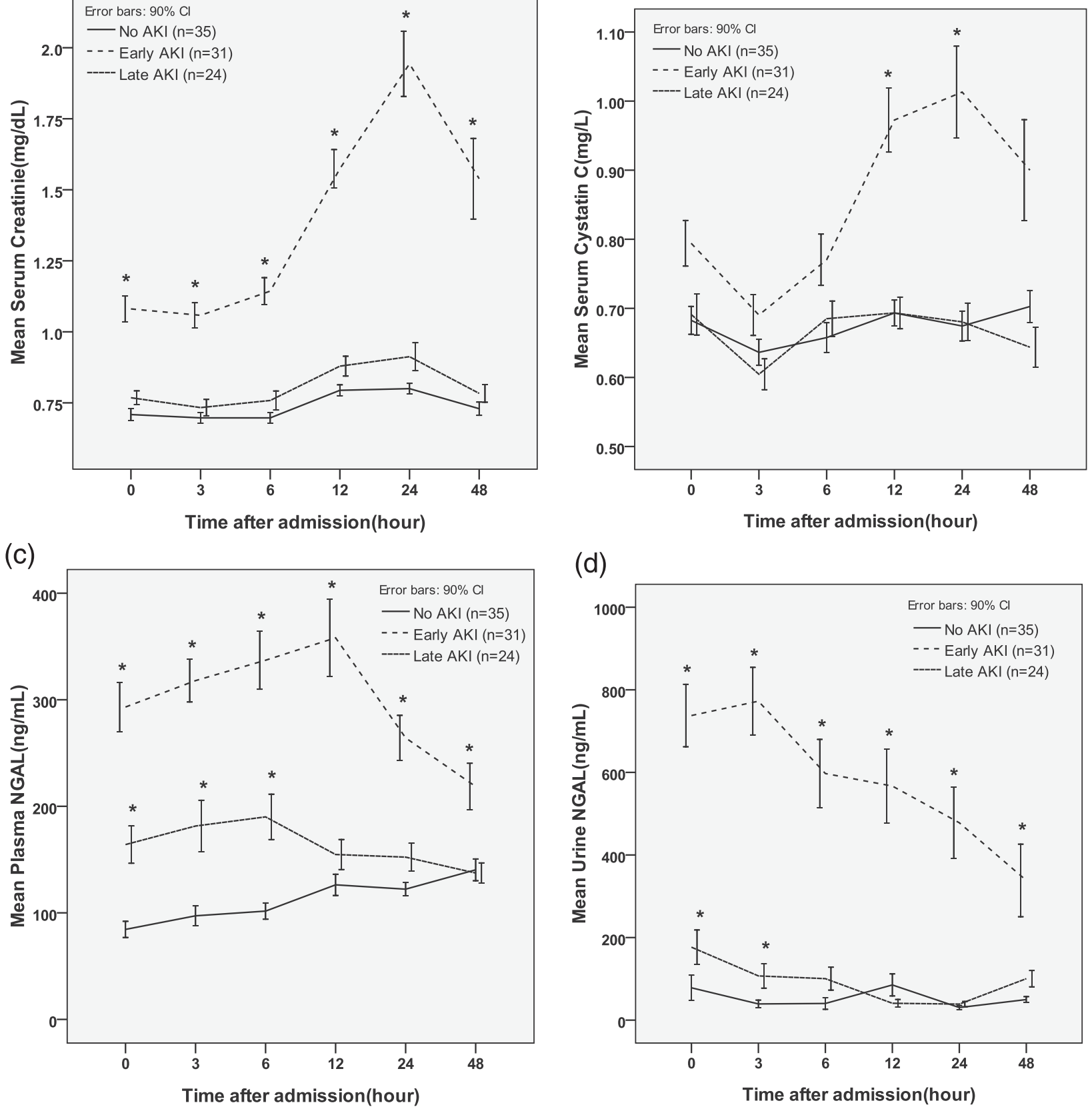

(d)

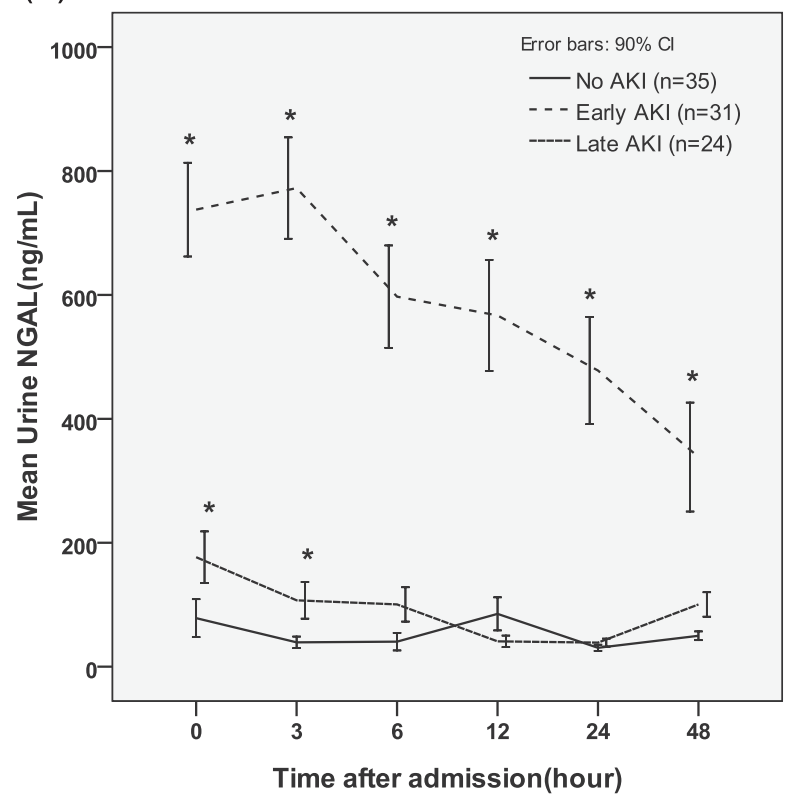

Figure 1 Comparison of serum creatinine (a), cystatin C (b), plasma neutrophil gelatinase-associated lipocalin (NGAL) (c), and urine NGAL (d) levels at different time points with respect to acute kidney injury (AKI) development. Early AKl, development of AKI within 5 days of injury; Late AKI: development of AKI beyond 5 days after injury. ${ }^{*}$ Significant difference $(P<0.05)$ relative to the no AKI group.

over time were compared between the groups (Figure 1). At all time points, the serum creatinine and plasma and urine NGAL levels were significantly higher in the early AKI group than in the non-AKI group. However, the cystatin $C$ levels were significantly higher only at 12 and $24 \mathrm{~h}$ after injury when comparing the early AKI and nonAKI groups.
To estimate the predictive power of each biomarker, we performed a ROC curve analysis for early AKI (Table 3(a)). The significant cut-off values for serum creatinine for predicting early AKI ranged from 0.85 to $1.15 \mathrm{mg} / \mathrm{dL}$ between the time points of 0 and $48 \mathrm{~h}$. The serum cystatin C levels were significant only at 12 and $24 \mathrm{~h}$, and the cut-off values ranged from 0.7 to $0.75 \mathrm{mg} / \mathrm{L}$. The 
(a)

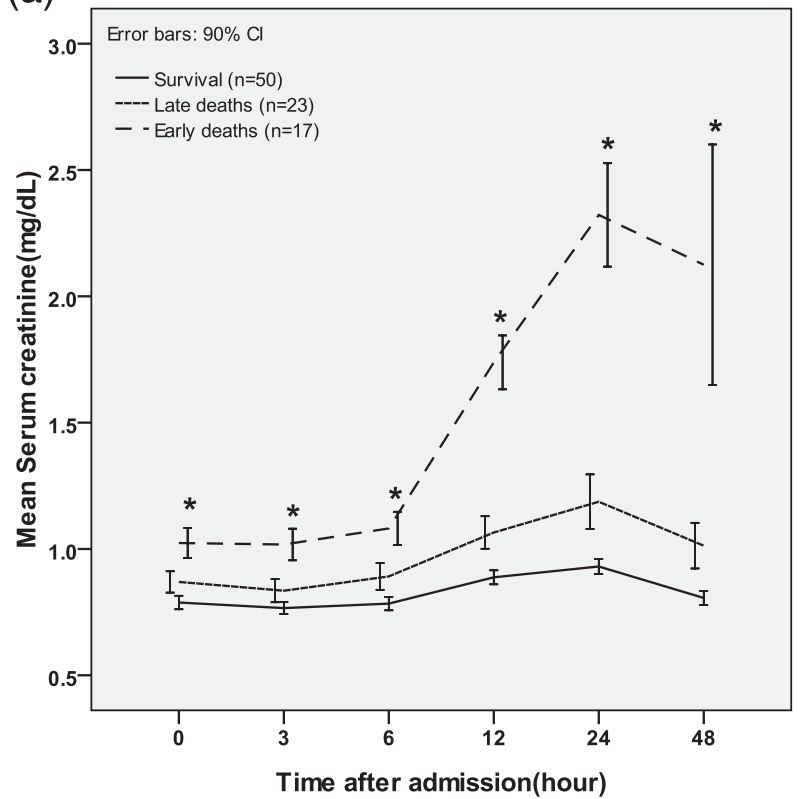

(c)

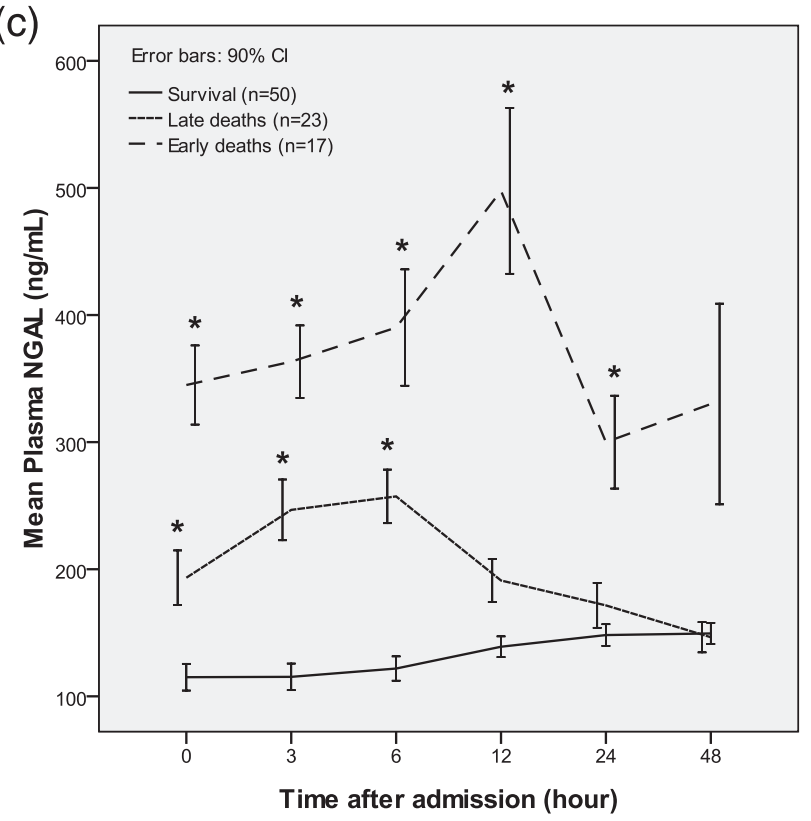

(b)

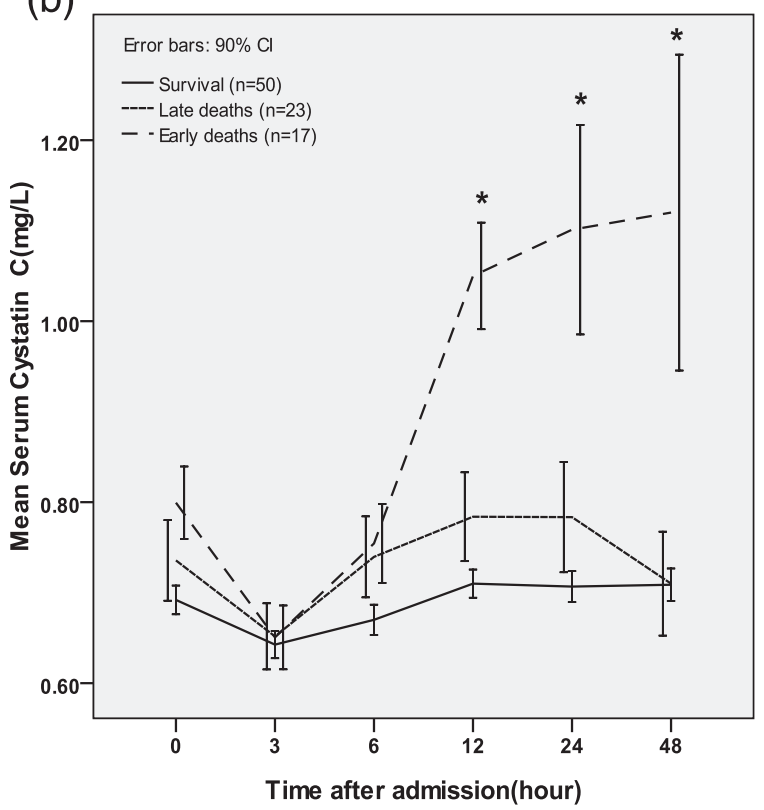

(d)

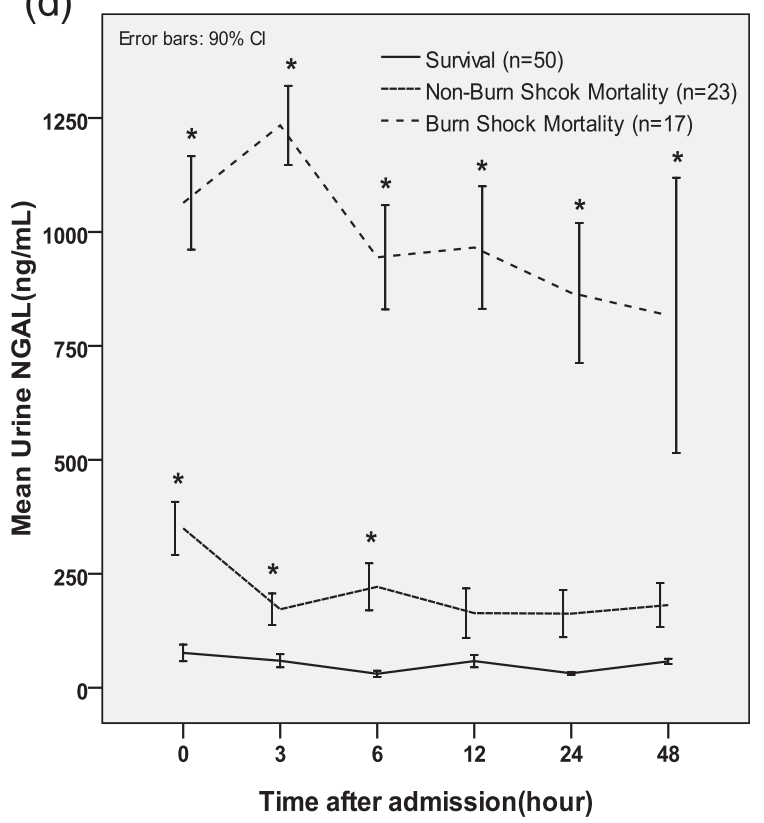

Figure 2 Comparison of serum creatinine (a), cystatin C (b), plasma neutrophil gelatinase-associated lipocalin (NGAL) (c), and urine NGAL (d) levels at different time points with respect to mortality. Early deaths: non-survivors who developed burn shock despite fluid resuscitation, and died within 3 days of burn injury. Late deaths: mortality due to causes other than burn shock beyond 3 days after burn injury. *Significant difference $(P<0.05)$ relative to the survival group.

plasma NGAL cut-off values for predicting early AKI ranged from 100.5 to $185.5 \mathrm{ng} / \mathrm{mL}$ and the urine NGAL cut-off values ranged from 26.8 to $52.9 \mathrm{ng} / \mathrm{mL}$.

When we compared the non-AKI and late AKI groups with respect to each biomarker, the serum creatinine and cystatin $\mathrm{C}$ levels did not differ at any time point. However, the plasma and urine NGAL levels differed significantly between the non-AKI and late AKI groups only during the hyper-acute period (plasma NGAL: 0, 3, and $6 \mathrm{~h}$ after injury; urine NGAL: 0 and $3 \mathrm{~h}$ after injury).

In the ROC curve analysis for predicting late AKI, only the plasma and urine NGAL levels remained significant for up to $6 \mathrm{~h}$ from injury (Table 3(b)). The cut-off values for plasma NGAL ranged from 100.0 to $125.0 \mathrm{ng} / \mathrm{mL}$ 

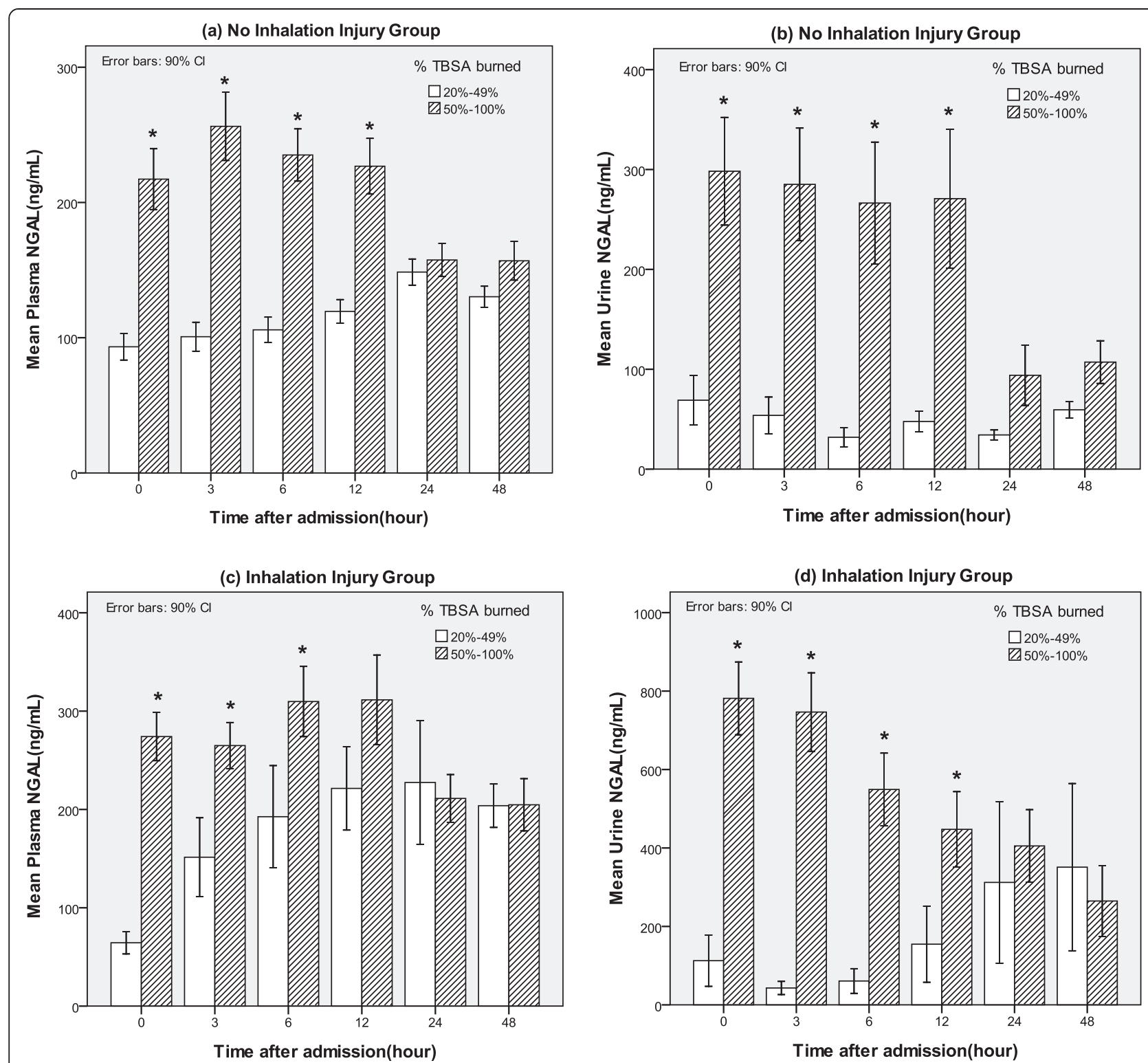

Figure 3 Comparison of the plasma and urine neutrophil gelatinase-associated lipocalin (NGAL) levels according to burn size and the presence of inhalation injuries. (a, b) No inhalation injury group. (c, d) Inhalation injury group. *Significant difference $(P<0.05)$ between groups. TBSA, total body surface area.

and the cut-off values for urine NGAL ranged from 7.25 to $11.95 \mathrm{ng} / \mathrm{mL}$.

\section{Changes in the levels of four biomarkers during $48 \mathrm{~h}$ after admission with respect to mortality}

The levels of each biomarker (serum creatinine, serum cystatin C, plasma NGAL, and urine NGAL) were compared between the groups classified according to mortality. At nearly all the time points, the serum creatinine, plasma NGAL, and urine NGAL levels were significantly higher in the early-death group than in the survivors group (Figure 2). However, the cystatin $\mathrm{C}$ levels were significantly higher in the early-death group only after $12 \mathrm{~h}$. In the ROC curve analysis for predicting early death, the cut-off values for serum creatinine ranged from 0.85 to $1.65 \mathrm{mg} / \mathrm{dL}$, those for plasma NGAL ranged from 144.5 to $191.5 \mathrm{ng} / \mathrm{mL}$ and those for urine NGAL ranged from 45.5 to $172.0 \mathrm{ng} / \mathrm{mL}$. The cut-off values for plasma NGAL ranged from 0.79 to $0.81 \mathrm{mg} / \mathrm{L}$ (Table $4(\mathrm{a})$ ).

When we compared the late-death and survivor groups with respect to each biomarker, no differences were found in the serum creatinine and cystatin $C$ levels at any time point. Only the plasma and urine NGAL levels differed significantly between the late death and survivor groups 

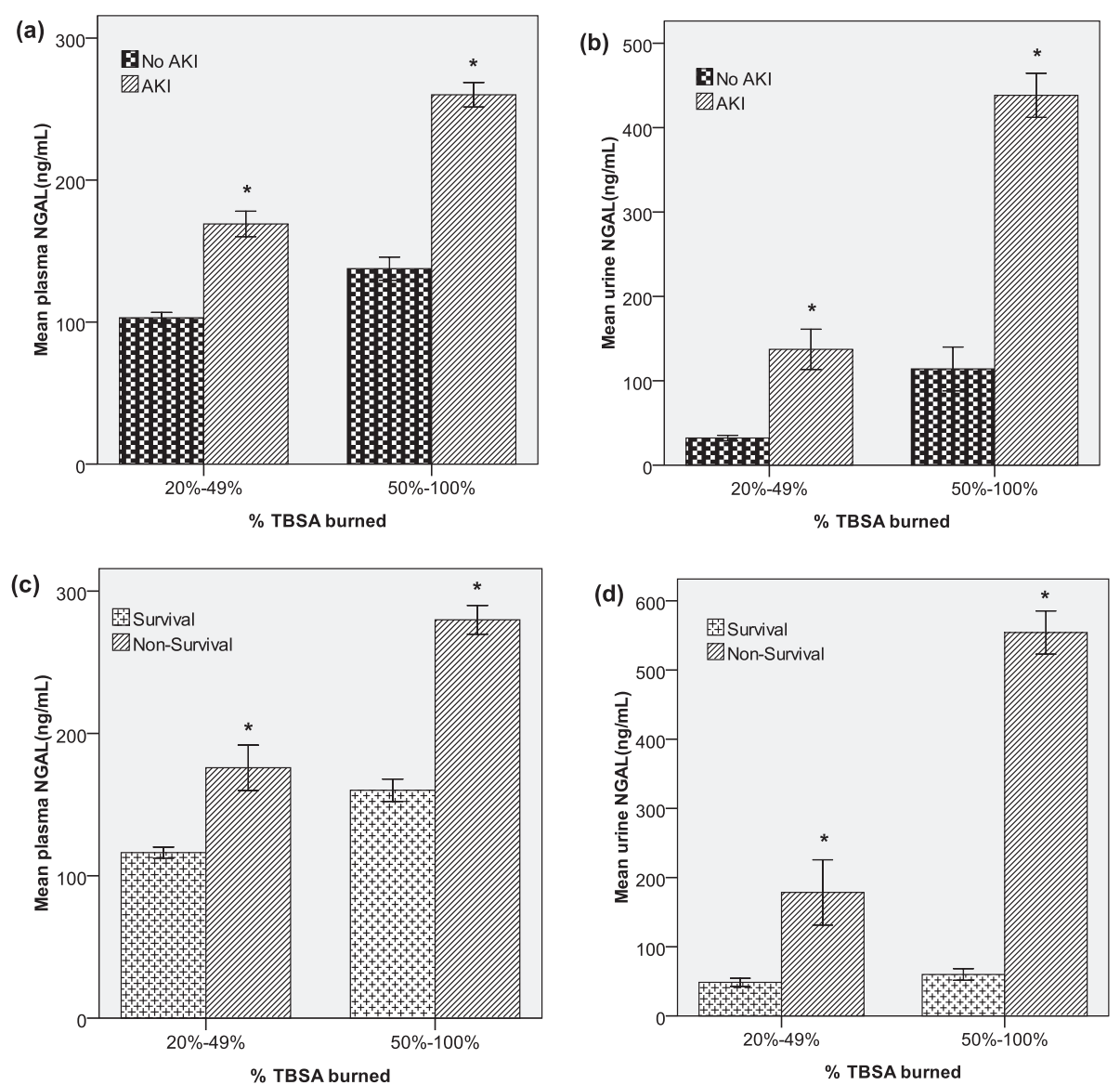

Figure 4 Comparison of the plasma and urine neutrophil gelatinase-associated lipocalin (NGAL) levels according to acute kidney injury and mortality at all time points and according to the subgroup for percentage total body surface area (TBSA) burned. (a, b) Acute kidney injury (AKI). (c, d) Mortality. *Significant difference $(P<0.05)$ between groups.

within $6 \mathrm{~h}$ of burn injury. In the ROC curve analysis for late death (Table 4(b)) the significant cut-off values for plasma NGAL ranged from 100.5 to $146.5 \mathrm{ng} / \mathrm{mL}$ within $6 \mathrm{~h}$ of admission. Additionally, the cut-off values for urine NGAL ranged from 15.6 to $20.1 \mathrm{ng} / \mathrm{mL}$ within $6 \mathrm{~h}$ of admission.

\section{Changes in plasma and urine NGAL levels with respect to burn size and inhalation injury}

The presence of inhalation injuries and the \% TBSA burned are the most powerful factors with which to determine the injury severity in major-burn patients. Therefore, we investigated the differences in the plasma and urine NGAL levels with respect to the injury severity as determined by the \% TBSA burned and the presence of inhalation injuries. According to our data, as the $\%$ TBSA burned increased (larger burn-size group: 50 to $100 \%$ TBSA burned), both the mean plasma NGAL and urine NGAL levels significantly increased within $12 \mathrm{~h}$ of admission in both patients with and without inhalation injuries (Figure 3).
As shown in Figure 3, we observed gross difference in plasma and urine NGAL with inhalation injury. In the patients with TBSA burned between 20 and 49\%, mean plasma NGAL was 116.1 in the patients without inhalation injury and 176.8 in those with inhalation injury $(P=0.018)$. Also, mean urine NGAL in these patients was significantly different with inhalation injury (49.4 without inhalation injury versus 172.4 with inhalation injury, $P=0.048)$. In the patients with TBSA burned between 50 and $100 \%$, there were significant differences in plasma NGAL (211.1 without inhalation injury versus 267.5 with inhalation injury, $P=0.013$ ) and urine NGAL (227.0 without inhalation injury versus 556.3 with inhalation injury, $P=0.000$ ) by the presence of inhalation injury.

After classifying the patients into subgroups according to $50 \%$ TBSA burned, we investigated changes in the plasma and urine NGAL levels with respect to AKI and mortality. In both groups ( $<50 \%$ and $\geq 50 \%$ TBSA burned), the plasma NGAL levels differed significantly with respect to AKI (no AKI versus AKI; $<50 \%$ burned, 103.0 versus $169.1 \mathrm{ng} / \mathrm{mL}$, 
Table 3 Performance characteristics of biochemical markers with for predicting acute kidney injury (AKI)

\begin{tabular}{|c|c|c|c|c|c|c|}
\hline \multicolumn{7}{|c|}{$\begin{array}{l}\text { (a) Performance characteristics of biochemical markers for } \\
\text { predicting early AKI development }(n=66)\end{array}$} \\
\hline & \multicolumn{6}{|c|}{ Time from admission } \\
\hline & $\mathrm{Oh}$ & $3 \mathrm{~h}$ & $6 \mathrm{~h}$ & $12 \mathrm{~h}$ & $24 \mathrm{~h}$ & $48 \mathrm{~h}$ \\
\hline AUC of serum creatinine & $0.816^{*}$ & $0.823^{*}$ & $0.881^{*}$ & $0.988^{*}$ & $0.987^{*}$ & $0.898^{*}$ \\
\hline Cut-off value, mg/dL & 0.85 & 0.85 & 0.85 & 1.05 & 1.15 & 0.95 \\
\hline Sensitivity & 0.710 & 0.645 & 0.700 & 0.926 & 0.913 & 0.778 \\
\hline Specificity & 0.706 & 0743 & 0.824 & 0.971 & 1.000 & 0.853 \\
\hline UC of serum cystatin $C$ & 0.615 & 0.54 & 0.605 & $0.746^{*}$ & $0.731^{*}$ & 0.619 \\
\hline Cut-off value, mg/L & & & & 0.70 & 0.75 & \\
\hline Sensit & & & & 0.704 & 0.652 & \\
\hline Specificity & & & & 0.500 & 0.618 & \\
\hline AUC of plasma NGAL & $0.832^{*}$ & $0.905^{*}$ & $0.926^{*}$ & $0.847^{*}$ & $0.818^{*}$ & $0.705^{*}$ \\
\hline Cut-off value, ng/mL & 100.5 & 172.0 & 159.0 & 175.5 & 185.5 & 144.0 \\
\hline Sensitivity & 0.806 & 0.806 & 0.900 & 0.815 & 0.739 & 0.667 \\
\hline Specificity & 0.735 & 0.800 & 0.794 & 0.765 & 0.912 & 0.647 \\
\hline UC of urine $N$ & $0.921^{*}$ & $0.927^{*}$ & $0.896^{*}$ & $0.813^{*}$ & $0.914^{*}$ & $0.802^{*}$ \\
\hline Cut-off value, ng/mL & 36.6 & 31.9 & 26.8 & 26.8 & 42.3 & 52.9 \\
\hline Sensitivity & 0.871 & 0.871 & 0.800 & 0.815 & 0.870 & 0.722 \\
\hline Specificity & 0.824 & 0.829 & 0.853 & 0.765 & 0.824 & 0.706 \\
\hline
\end{tabular}

(b) Performance characteristics of biochemical markers for predicting late AKI development $(n=59)$

Time from admission

\begin{tabular}{lllllll} 
& $\mathbf{0 ~ h}$ & $\mathbf{3} \mathbf{~ h}$ & $\mathbf{6} \mathbf{~ h}$ & $\mathbf{1 2} \mathbf{~ h}$ & $\mathbf{2 4} \mathbf{~ h}$ & $\mathbf{4 8} \mathbf{~ h}$ \\
\hline AUC of serum creatinine & 0.581 & 0.55 & 0.609 & 0.605 & $0.660^{*}$ & 0.625 \\
Cut-off value, mg/dL & & & & & 0.85 & \\
Sensitivity & & & & & 0.739 & \\
Specificity & & & & & 0.676 & \\
AUC of serum cystatin C & 0.488 & 0.437 & 0.545 & 0.491 & 0.513 & 0.444 \\
AUC of plasma NGAL & $0.682^{*}$ & $0.658^{*}$ & $0.670^{*}$ & 0.605 & 0.571 & 0.537 \\
Cut-off value, ng/mL & 102.5 & 100.0 & 125.0 & & & \\
Sensitivity & 0.640 & 0.583 & 0.625 & & & \\
Specificity & 0.735 & 0.629 & 0.676 & & & \\
AUC of urine NGAL & $0.692^{*}$ & $0.681^{*}$ & 0.644 & 0.599 & 0.598 & 0.604 \\
Cut-off value, ng/mL & 11.95 & 7.25 & 8.25 & & & \\
Sensitivity & 0.680 & 0.708 & 0.625 & & & \\
Specificity & 0.647 & 0.600 & 0.588 & & & \\
\hline
\end{tabular}

Early AKI, development of AKI within 5 days of injury; late AKI, development of AKI beyond 5 days after injury; AUC, area under the receiver operating characteristic curve; NGAL, neutrophil gelatinase-associated lipocalin. * Statistically significant difference $(P<0.05)$.

$P<0.001 ; \geq 50 \%$ burned, 137.6 versus $260.0 \mathrm{ng} / \mathrm{mL}$, $P<0.001)$. Similarly, the urine NGAL levels differed significantly with respect to AKI ( $<50 \%$ burned, 32.1 versus $137.2 \mathrm{ng} / \mathrm{mL}, P<0.001 ; \geq 50 \%$ burned, 114.0 versus $438.2 \mathrm{ng} / \mathrm{mL}, P<0.001$; Figure $4(\mathrm{a}), 4(\mathrm{~b}))$. In the same
Table 4 Performance characteristics of biochemical markers for predicting mortality

\section{(a) Performance characteristics of biochemical markers for} predicting early death $(n=67)$

\begin{tabular}{cllllll} 
& \multicolumn{7}{c}{ Time from admission } & & \\
\cline { 2 - 7 } & $\mathbf{0 ~ h}$ & $\mathbf{3} \mathbf{h}$ & $\mathbf{6} \mathbf{~ h}$ & $\mathbf{1 2} \mathbf{~ h}$ & $\mathbf{2 4} \mathbf{~ h}$ & $\mathbf{4 8} \mathbf{~ h}$ \\
\hline AUC of serum creatinine & $0.699^{*}$ & $0.697^{*}$ & $0.747^{*}$ & $0.960^{*}$ & $0.941^{*}$ & $0.946^{*}$ \\
Cut-off value, mg/dL & 0.85 & 0.85 & 0.85 & 1.15 & 1.65 & 0.95 \\
Sensitivity & 0.588 & 0.529 & 0.625 & 0.923 & 0.889 & 1.000 \\
Specificity & 0.640 & 0.640 & 0.694 & 0.898 & 0.980 & 0.776 \\
AUC of serum cystatin C & 0.636 & 0.501 & 0.581 & $0.812^{*}$ & $0.757^{*}$ & $0.811^{*}$ \\
Cut-off value(mg/L) & & & & 0.80 & 0.81 & 0.79 \\
Sensitivity & & & & 0.692 & 0.667 & 0.750 \\
Specificity & & & & 0.694 & 0.735 & 0.714 \\
AUC of plasma NGAL & $0.848^{*}$ & $0.919^{*}$ & $0.920^{*}$ & $0.892^{*}$ & $0.804^{*}$ & 0.776 \\
Cut-off value, ng/mL & 144.5 & 191.5 & 181.0 & 177.0 & 185.5 & \\
Sensitivity & 0.824 & 0.882 & 0.938 & 0.923 & 0.778 & \\
Specificity & 0.760 & 0.820 & 0.837 & 0.702 & 0.735 & \\
AUC of urine NGAL & $0.953^{*}$ & $0.984^{*}$ & $0.967^{*}$ & $0.940^{*}$ & $0.972^{*}$ & $0.939^{*}$ \\
Cut-off value, ng/mL & 98.9 & 172.0 & 45.5 & 46.0 & 50.0 & 83.8 \\
Sensitivity & 0.941 & 0.941 & 1.000 & 1.000 & 1.000 & 1.000 \\
Specificity & 0.840 & 0.920 & 0.878 & 0.796 & 0.878 & 0.796 \\
\hline
\end{tabular}

(b) Performance characteristics of biochemical markers for predicting late death $(n=73)$

\begin{tabular}{cllllll} 
& \multicolumn{7}{c}{ Time from admission } \\
\cline { 2 - 7 } & $\mathbf{0} \mathbf{h}$ & $\mathbf{3} \mathbf{h}$ & $\mathbf{6} \mathbf{~ h}$ & $\mathbf{1 2} \mathbf{~ h}$ & $\mathbf{2 4} \mathbf{~ h}$ & $\mathbf{4 8} \mathbf{~}$ \\
\hline AUC of serum creatinine & 0.595 & 0.561 & 0.609 & 0.625 & 0.594 & 0.617 \\
AUC of serum cystatin C & 0.478 & 0.450 & 0.529 & 0.503 & 0.463 & 0.425 \\
AUC of plasma NGAL & $0.650^{*}$ & $0.765^{*}$ & $0.800^{*}$ & 0.629 & 0.534 & 0.515 \\
Cut-off value. ng/mL & 100.5 & 126.5 & 146.5 & & & \\
Sensitivity & 0.696 & 0.739 & 0.783 & & & \\
Specificity & 0.600 & 0.700 & 0.714 & & & \\
AUC of urine NGAL & $0.734^{*}$ & $0.691^{*}$ & $0.717^{*}$ & 0.607 & 0.635 & 0.583 \\
Cut-off value, ng/mL & 20.1 & 16.7 & 15.6 & & & \\
Sensitivity & 0.696 & 0.696 & 0.739 & & & \\
Specificity & 0.640 & 0.640 & 0.673 & & & \\
\hline
\end{tabular}

Early death, non-survivors who developed burn shock despite fluid resuscitation and died within 3 days of burn injury; late death, mortality due to causes other than burn shock beyond 3 days; AUC, area under the receiver operating characteristic curve; NGAL, neutrophil gelatinase-associated lipocalin. *Statistically significant difference $(P<0.05)$.

subgroups, the plasma NGAL levels differed significantly with respect to mortality (survivors versus nonsurvivors $<50 \%$ burned, 116.2 versus $172.9 \mathrm{ng} / \mathrm{mL}, P=$ 0.018 ; $\geq 50 \%$ burned, 117.3 versus $201.8 \mathrm{ng} / \mathrm{mL}, P<0.001$ ). Similarly, the urine NGAL levels differed significantly according to mortality (<50\% burned, 48.5 versus $178.5 \mathrm{ng} /$ $\mathrm{mL}, P=0.049 ; \geq 50 \%$ burned, 59.9 versus $554.1 \mathrm{ng} / \mathrm{mL}$, $P<0.001$; Figure 4(c), (d)). 
Table 5 Multivariate binary logistic regression analysis for acute kidney injury (AKI) development and mortality at all time points within $48 \mathrm{~h}$ of admission

(a) Multivariate logistic regression analysis of AKI development

\begin{tabular}{llllll} 
& & & \multicolumn{2}{c}{$\mathbf{9 5 \%} \mathbf{C l}$ for } \\
& & & & \multicolumn{2}{c}{ odds ratio } \\
\cline { 5 - 6 } & Odds ratio & $P$-value & Lower & Upper \\
\hline Age $>60$ years & & & 3.107 & 12.117 \\
TBSA burned $>50 \%$ & 8.628 & $0.000^{*}$ & 5.036 & 14.783 \\
Sex, female & 4.199 & $0.000^{*}$ & 1.940 & 9.086 \\
Inhalation injury present & 3.129 & $0.000^{*}$ & 1.734 & 5.647 \\
Serum creatinine $>1.1 \mathrm{mg} / \mathrm{dL}$ & 74.930 & $0.000^{*}$ & 7.926 & 708.331 \\
Serum cystatin C $>1.1 \mathrm{mg} / \mathrm{L}$ & 0.150 & $0.038^{*}$ & 0.025 & 0.902 \\
Plasma NGAL $>153 \mathrm{ng} / \mathrm{mL}$ & 1.924 & $0.016^{*}$ & 1.128 & 3.282 \\
Urine NGAL $>131 \mathrm{ng} / \mathrm{mL}$ & 2.568 & $0.009^{*}$ & 1.263 & 5.220 \\
\hline
\end{tabular}

(b) Multivariate logistic regression analysis of mortality

\begin{tabular}{|c|c|c|c|c|}
\hline & \multirow[b]{2}{*}{ Odds ratio } & \multirow[b]{2}{*}{$P$-value } & \multicolumn{2}{|c|}{$\begin{array}{l}95 \% \mathrm{Cl} \text { for } \\
\text { odds ratio }\end{array}$} \\
\hline & & & Lower & Upper \\
\hline Age $>60$ years & 23.322 & $0.000^{*}$ & 9.885 & 55.023 \\
\hline TBSA burned >50\% & 25.821 & $0.000^{*}$ & 12.037 & 55.389 \\
\hline Sex, female & 4.704 & $0.000^{*}$ & 1.999 & 11.070 \\
\hline Inhalation injury present & 0.923 & 0.771 & 0.536 & 1.588 \\
\hline Serum creatinine $>1.1 \mathrm{mg} / \mathrm{dL}$ & 0.976 & 0.947 & 0.480 & 1.986 \\
\hline Serum cystatin $C>1.1 \mathrm{mg} / \mathrm{L}$ & 2.562 & 0.137 & 0.741 & 8.853 \\
\hline Plasma NGAL >153 ng/mL & 1.886 & $0.023^{*}$ & 1.091 & 3.259 \\
\hline Urine NGAL >131 ng/mL & 7.854 & $0.000^{*}$ & 3.949 & 15.622 \\
\hline
\end{tabular}

The biomarker cut-off values were designated according to normal reference ranges, regardless of our results. The model was based on the univariate analysis, and no significant interactions between correlated variables were found in a correlation matrix. ${ }^{*}$ Statistically significant difference $(P<0.05)$.

\section{Multivariate logistic regression analysis for predicting AKI} and mortality

A multivariate binary logistic regression analysis for predicting AKI and mortality was performed and included the laboratory results at all time points. The age groups were categorized as either $<60$ or $\geq 60$ years, the $\%$ TBSA burned groups as $<50 \%$ or $\geq 50 \%$ of $\%$ TBSA burned, the serum creatinine levels as $<1.1$ or $\geq 1.1 \mathrm{mg} / \mathrm{dL}$, the serum cystatin C levels as $<1.1$ or $\geq 1.1 \mathrm{mg} / \mathrm{L}$, the plasma NGAL levels as $<153$ or $\geq 153 \mathrm{ng} / \mathrm{mL}$, and the urine NGAL levels as $<131$ or $\geq 131 \mathrm{ng} / \mathrm{mL}$. The cut-off values of the biomarkers were determined according to normal reference ranges from non-hospitalized donors. The analysis model was based on the univariate analysis, and no significant interactions were found between correlated variables in a correlation matrix.

All variables, including age, \% TBSA burned, sex, inhalation injury, serum creatinine levels, serum cystatin $\mathrm{C}$ levels, plasma NGAL levels, and urine NGAL levels, were independently associated with AKI development
(Table 5(a)). Age, sex, \% TBSA burned, plasma NGAL levels, and urine NGAL levels were also independently associated with mortality. However, inhalation injury, serum creatinine levels, and serum cystatin $C$ levels were not independently associated with mortality (Table 5(b)).

\section{Discussion}

In this study, we evaluated the diagnostic utility of cystatin C, plasma NGAL, and urine NGAL levels in the early post-burn period for predicting AKI and mortality in patients with major burn injuries. In massively burned patients, the mechanisms associated with early AKI development and early death differ considerably from the mechanisms associated with late AKI development and late death. Therefore, this analysis evaluated both early and late AKI and both early and late deaths.

All four markers (serum creatinine, serum cystatin C, plasma NGAL, and urine NGAL) were useful for predicting early AKI and early deaths at nearly all the investigated time points (Figures 1 and 2). However, the serum creatinine and cystatin C levels rapidly increased only after $12 \mathrm{~h}$ from admission. In contrast, the plasma and urine NGAL levels had already increased rapidly at the time of admission. During early AKI in massively burned patients, the urine NGAL levels first increased, followed by the plasma NGAL, cystatin C, and serum creatinine levels. Based on these results, we suspected that serum creatinine could not be used to diagnose the hyper-acute stage of AKI and that the plasma and urine NGAL levels were superior biochemical markers for diagnosing AKI. However, in patients with larger burn-wound surface areas, we observed increases in both the plasma and urine NGAL levels, as shown in Figure 3. This indicates that the \% TBSA burned is a possible confounding factor for predictions of early AKI and early death. However, even in the subgroups classified according to the \% TBSA burned values $(<50 \%$ or $\geq 50 \%$ ), the plasma and urine NGAL levels differed significantly with respect to AKI and mortality (Figure 4).

Only the plasma and urine NGAL levels exhibited statistical significance for predicting late AKI and late deaths within $6 \mathrm{~h}$ of admission. However, given these results, we cannot confirm that the plasma and urine NGAL levels can distinguish those patients who will develop late AKI and experience late death from those who will not develop AKI and will survive. The mean \% TBSA burned values were $38.4 \% \pm 14.1 \%$ in the no-AKI group versus $61.5 \% \pm 19.2 \%$ in the late-AKI group and $41.9 \% \pm$ $15.2 \%$ in the survivor group versus $65.7 \% \pm 18.4 \%$ in the late-death group. All of the AUCs for the plasma and urine NGAL levels within $6 \mathrm{~h}$ were $<0.7$ for late AKI and late death. Therefore, we cannot confirm that the plasma and urine NGAL levels can predict late AKI development and late death in massively burned patients because of the possible confounding effect of the burn 
surface area. The cut-off values for urine NGAL were considerably lower than the normal reference range (Tables 3 and 4), suggesting that patients with intact renal function experienced significant diuresis due to a massive fluid resuscitation. Therefore, these cut-off values are not necessarily transferable to other situations.

In a multivariate logistic regression analysis that included the laboratory results at all time points, all the variables - including age, \% TBSA burned, sex, presence of inhalation injury, and serum creatinine, serum cystatin C, plasma NGAL, and urine NGAL levels - were independently associated with AKI development (Table 5(a)). Age, sex, \% TBSA burned, plasma NGAL levels, and urine NGAL levels were also independently associated with mortality. However, inhalation injury, serum creatinine levels, and serum cystatin $C$ levels were not independently associated with mortality (Table 5(b)). Based on these results, we can confirm that the plasma and urine NGAL levels in the early post-burn period can predict AKI and mortality in major-burn patients. Our results are consistent with two recent reports demonstrating NGAL as an indicator for AKI in burn patients [14,15].

A limitation of this study is that early AKI was already present at admission, and therefore, the utility of refining the AKI diagnosis is unclear. The very good diagnostic performance of the creatinine levels at 12 to $24 \mathrm{~h}$ is unsurprising, as this test represents the gold standard for early AKI diagnosis. However, the poor gold standard for AKI assessment limits the performance characteristics of even the best biomarkers.

\section{Conclusions}

Massively burned patients who maintain high plasma and urine NGAL levels until $48 \mathrm{~h}$ after admission are at risk of early AKI development and early mortality with burn shock. However, the plasma and urine NGAL levels in the early post-burn period failed to predict late AKI development and late death in this study. Nonetheless, the plasma and urine NGAL levels within $48 \mathrm{~h}$ of admission were independently associated with AKI development and mortality. In a future study, we should further investigate the diagnostic efficacy of plasma and urine NGAL levels in massively burned patients during the late post-burn period with regard to AKI and mortality.

\section{Key messages}

- In massively burned patients, the plasma and urine NGAL levels increased rapidly during early AKI, followed by the cystatin $\mathrm{C}$ and serum creatinine levels.

- Increased plasma and urine NGAL levels in the early post-burn period were associated with early
AKI and burn shock mortality but failed to predict late AKI and non-burn shock mortality.

- The plasma and urine NGAL levels increased significantly in patients with larger burn-wound surface areas.

- The plasma and urine NGAL levels within $48 \mathrm{~h}$ of admission were independently associated with AKI development and mortality.

\section{Abbreviations}

AKl: acute kidney injury; AUC: area under the curve; BS: burn shock; CB: chemical burn; Cr: Creatinine; CRRT: continuous renal replacement therapy; EB: electrical burn; FB: flame burn; GFR: glomerular filtration rate; LOS: length of hospital stay; MD: medication; NGAL: neutrophil gelatinaseassociated lipocalin; RD: rhabdomyolysis; RIFLE: risk injury, failure, sustained loss, and end-stage kidney disease; ROC: receiver operating characteristic; SB: scalding burn; SKB: spark burn; SS: septic shock; TBSA: total body surface area.

\section{Competing interests}

The authors declare that they have no competing interests.

\section{Authors' contributions}

HTY and HSK developed the study concept and design. HY, YSC, DK, JH, JHK, and WC performed the acquisition of data and analysis and the interpretation of the data. HTY and HSK wrote the manuscript. All authors read and approved the final manuscript for publication.

\section{Acknowledgements}

We appreciate Abbott Diagnostics for providing the urine NGAL reagents, and appreciate Alere Healthcare for providing Triage NGAL reagent. We also thank Hye Won Kwon, Ki Young Kwon, and Hee Sun Park for excellent technical assistance and collection of specimens and data.

\section{Author details}

${ }^{1}$ Department of Surgery, Burn center, Hallym University Hangang Sacred Heart Hospital, Hallym University College of Medicine, 12, Beodeunaru-ro 7-gil, Youngdeungpo-gu, Seoul 150-719, Korea. ${ }^{2}$ Department of Laboratory Medicine, Hallym University Dongtan Sacred Heart Hospital, Hallym University College of Medicine, 7, Keunjaebong-gil, Hwaseong-si, Gyeonggi-do 445-170, Korea. ${ }^{3}$ Department of Surgery, Kangwon National University College of Medicine, 1 Kangwondaehak-gil, Chuncheon-si, Gangwon-do 200-701, Korea.

Received: 3 December 2013 Accepted: 24 June 2014

Published: 14 July 2014

\section{References}

1. Holm C, Hörbrand F, von Donnersmarck GH, Mühlbauer W: Acute renal failure in severely burned patients. Burns 1999, 25:171-178.

2. Chrysopoulo MT, Jeschke MG, Dziewulski P, Barrow RE, Herndon DN: Acute renal dysfunction in severely burned adults. J Trauma 1999, 46:141-144.

3. Bellomo R, Kellum JA, Ronco C: Defining acute renal failure: physiological principles. Intensive Care Med 2004, 30:33-37.

4. Haase-Fielitz A, Bellomo R, Devarajan P, Story D, Matalanis G, Dragun D, Haase M: Novel and conventional serum biomarkers predicting acute kidney injury in adult cardiac surgery-a prospective cohort study. Crit Care Med 2009, 37:553-560.

5. Devarajan P: Emerging biomarkers of acute kidney injury. Contrib Nephrol 2007, 156:203-212.

6. Bökenkamp A, Domanetzki M, Zinck R, Schumann G, Byrd D, Brodehl J: Cystatin $\mathrm{C}$-a new marker of glomerular filtration rate in children independent of age and height. Pediatrics 1998, 101:875-881.

7. Mangge H, Liebmann P, Tanil H, Herrmann J, Wagner C, Gallistl S, Schauenstein K, Erwa W: Cystatin C, an early indicator for incipient renal disease in rheumatoid arthritis. Clin Chim Acta 2000, 300:195-202.

8. Finney $H$, Newman DJ, Gruber W, Merle P, Price CP: Initial evaluation of cystatin $C$ measurement by particle-enhanced immunonephelometry 
on the Behring nephelometer systems (BNA, BN II). Clin Chem 1997, 43:1016-1022

9. Newman DJ, Thakkar H, Edwards RG, Wilkie M, White T, Grubb AO, Price CP: Serum cystatin $C$ measured by automated immunoassay: a more sensitive marker of changes in GFR than serum creatinine. Kidney Int 1995, 47:312-318.

10. Mori K, Nakao K: Neutrophil gelatinase-associated lipocalin as the real-time indicator of active kidney damage. Kidney Int 2007, 71:967-970.

11. Mori K, Lee HT, Rapoport D, Drexler IR, Foster K, Yang J, Schmidt-Ott KM, Chen X, Li JY, Weiss S, Mishra J, Cheema FH, Markowitz G, Suganami T, Sawai K, Mukoyama M, Kunis C, D'Agati V, Devarajan P, Barasch J: Endocytic delivery of lipocalin-siderophore-iron complex rescues the kidney from ischemia-reperfusion injury. J Clin Invest 2005, 115:610-621.

12. Mishra J, Ma Q, Prada A, Mitsnefes M, Zahedi K, Yang J, Barasch J, Devarajan P. Identification of neutrophil gelatinase-associated lipocalin as a novel early urinary biomarker for ischemic renal injury. J Am Soc Nephrol 2003, 14:2534-2543

13. Bellomo R, Ronco C, Kellum JA, Mehta RL, Palevsky P, workgroup ADQI: Acute renal failure - definition, outcome measures, animal models, fluid therapy and information technology needs: the Second International Consensus Conference of the Acute Dialysis Quality Initiative (ADQI) Group. Crit Care 2004, 8:R204-R212.

14. Hong DY, Lee JH, Park SO, Baek KJ, Lee KR: Plasma neutrophil gelatinaseassociated lipocalin (NGAL) as a early biomarker for acute kidney injury in burn patients. J Burn Care Res 2013, 34:e326-e332.

15. Yavuz S, Anarat A, Acartürk S, Dalay AC, Kesiktas E, Yavuz M, Acartürk TO: Neutrophil gelatinase associated lipocalin as an indicator of acute kidney injury and inflammation in burn children. Burns 2014, 40:648-654.

doi:10.1186/cc13989

Cite this article as: Yang et al: Assessment of biochemical markers in the early post-burn period for predicting acute kidney injury and mortality in patients with major burn injury: comparison of serum creatinine, serum cystatin- $C$, plasma and urine neutrophil gelatinaseassociated lipocalin. Critical Care 2014 18:R151.

\section{Submit your next manuscript to BioMed Central and take full advantage of:}

- Convenient online submission

- Thorough peer review

- No space constraints or color figure charges

- Immediate publication on acceptance

- Inclusion in PubMed, CAS, Scopus and Google Scholar

- Research which is freely available for redistribution 